\title{
Two-dimensional Kagome photonic bandgap waveguide
}

Nielsen, Jens Bo; Søndergaard, Thomas; Libori, Stig E. Barkou; Bjarklev, Anders Overgaard; Broeng, Jes

Published in:

I E E E Photonics Technology Letters

Link to article, DOI:

$10.1109 / 68.849066$

Publication date:

2000

Document Version

Publisher's PDF, also known as Version of record

Link back to DTU Orbit

Citation (APA):

Nielsen, J. B., Søndergaard, T., Libori, S. E. B., Bjarklev, A. O., \& Broeng, J. (2000). Two-dimensional Kagome photonic bandgap waveguide. I E E E Photonics Technology Letters, 12(6), 630-632.

https://doi.org/10.1109/68.849066

\section{General rights}

Copyright and moral rights for the publications made accessible in the public portal are retained by the authors and/or other copyright owners and it is a condition of accessing publications that users recognise and abide by the legal requirements associated with these rights.

- Users may download and print one copy of any publication from the public portal for the purpose of private study or research.

- You may not further distribute the material or use it for any profit-making activity or commercial gain

- You may freely distribute the URL identifying the publication in the public portal

If you believe that this document breaches copyright please contact us providing details, and we will remove access to the work immediately and investigate your claim 


\title{
Two-Dimensional Kagomé Photonic Bandgap Waveguide
}

\author{
Jens Bo Nielsen, Thomas Søndergaard, Stig E. Barkou, Anders Bjarklev, and Jes Broeng
}

\begin{abstract}
The transverse-magnetic photonic-bandgap-guidance properties are investigated for a planar two-dimensional (2-D) Kagomé waveguide configuration using a full-vectorial plane-wave-expansion method. Single-moded well-localized low-index guided modes are found. The localization of the optical modes is investigated with respect to the width of the 2-D Kagomé waveguide, and the number of modes existing for specific frequencies and waveguide widths is mapped out.
\end{abstract}

Index Terms-Integrated optics, Kagomé lattice, periodic structures, photonic bandgap, submillimeter wave waveguides.

I N RECENT YEARS, a new class of periodic dielectric structures, known as photonic bandgap structures, has been discovered [1]. These structures are characterized by one or more frequency intervals, photonic bandgaps, where propagation of electromagnetic waves is not allowed. By introducing a properly designed defect in a photonic bandgap structure strong localization of photon waves to the region near the defect is possible. A novel microcavity design may be obtained by introducing a point defect, whereas novel waveguide designs may be obtained by introducing a line defect. A recent theoretical result for a waveguide based on introducing a line defect in a two-dimensional (2-D) photonic bandgap structure showed that a transmission of $98 \%$ around a $90^{\circ}$ sharp bend is possible [2]. The most commonly known 2-D photonic bandgap structures are the square lattice structure [3], [4], the triangular structure [5], [6], and the Honeycomb structure [7], [8]. It is a technological challenge to fabricate photonic bandgap structures for optical wavelengths, since the lattice constant of the periodic structure must be comparable to the optical wavelength. For structures based on GaAs rods in air, one approach is to use selective epitaxy [9].

In this letter, a waveguide based on a third fundamental hexagonal photonic bandgap structure, known as the Kagomé structure [10], is investigated for the first time. This waveguide, shown in Fig. 1 along with the coordinate system, consists of a homogeneous layer, the line defect, sandwiched between two semi-infinite layers of the Kagomé structure with circular dielectric rods in air. The parameters defining the 2-D waveguide structure are the background dielectric constant $\varepsilon_{\text {back }}=1$ (air), the dielectric constant of the rods $\varepsilon_{\text {rod }}=13$ (representative of GaAs at optical frequencies), the rod diameter $D$, the center-to-center distance between rods $\Lambda$,

Manuscript received November 4, 1999. This work was supported by the Danish Technical Research Council under the Technology by Highly Oriented Research (THOR) program.

The authors are with Research Center COM, Technical University of Denmark, DK-2800 Lyngby, Denmark (e-mail: ab@com.dtu.dk).

Publisher Item Identifier S 1041-1135(00)04599-7.

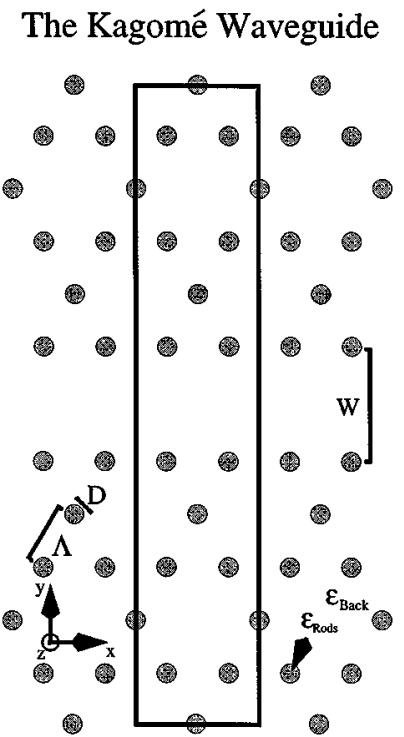

Fig. 1. The structure of the Kagomé waveguide is shown, along with an example of a supercell and the parameters necessary to characterize the structure.

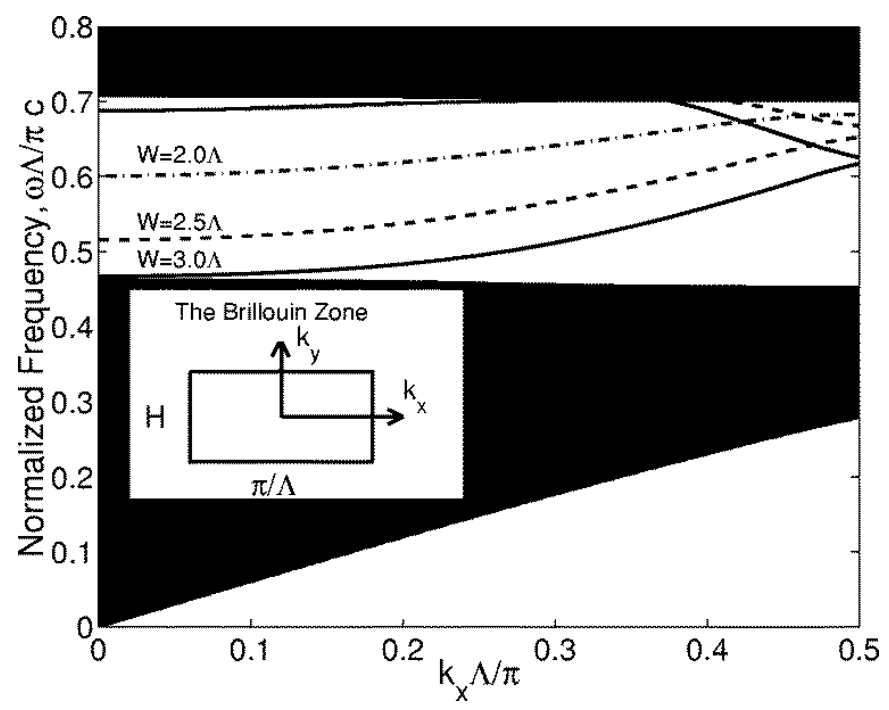

Fig. 2. TM band diagram of the Kagomé waveguide with localized modes for three different waveguide widths.

and the center-to-center waveguide width $W$. The structure is invariant in the out-of-plane direction (the $z$-direction).

The results presented in this letter were calculated using a computer model based on the full-vectorial plane-wave-expansion method described in [11]. In the calculations the Kagomé waveguide is approximated by a periodic structure using 


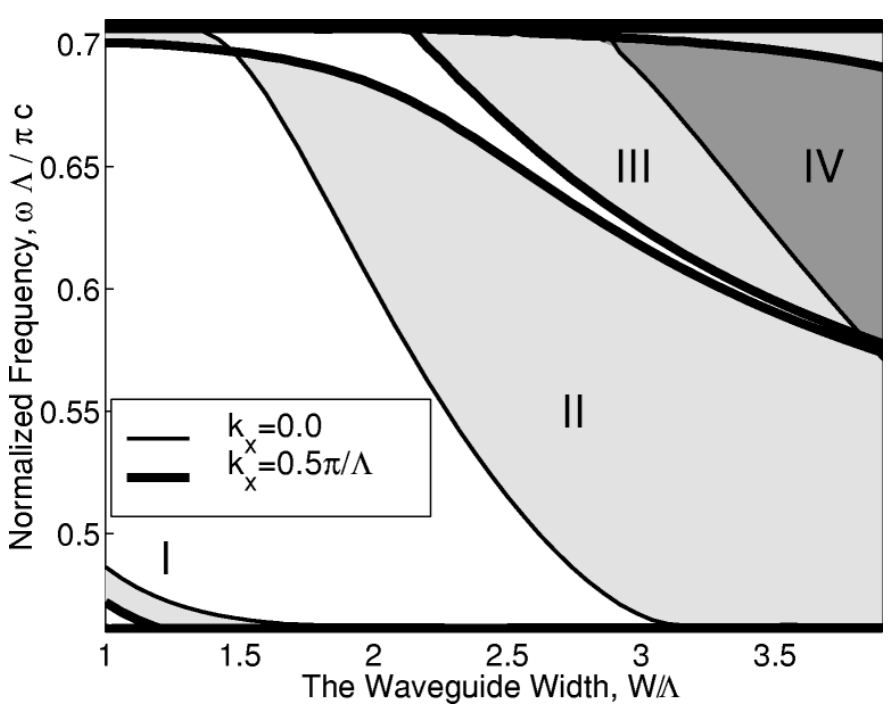

Fig. 3. The frequency domain of modes for small waveguide widths. Single-moded and dual-moded domains are colored light and dark, respectively. The thin domain edge corresponds to $k_{x}=0$, while the thick edge corresponds to $k_{x}=\pi / 2 \Lambda$.

a supercell approximation. An example of a supercell of height $H=W+5 \sqrt{3} \Lambda$ is shown in Fig. 1. In the actual calculations, a supercell of height $H=W+9 \sqrt{3} \Lambda$ was used, except for Fig. 3, where a supercell of height $H=W+17 \sqrt{3} \Lambda$ was used to test the convergence of the calculations. The differences in frequency between the calculations with supercell height $H=W+9 \sqrt{3} \Lambda$ and $H=W+17 \sqrt{3} \Lambda$ were smaller than $0.6 \%$.

For in-plane investigations it is common to categorize the modes in TE and TM modes defined as the modes having their electric and magnetic fields, respectively, in the plane of investigation (the $x y$-plane). In this letter, we will restrict our attention to TM polarized modes, and therefore choose the rod diameter $D=0.45 \Lambda$ yielding the largest TM photonic bandgap for the Kagomé rod structure with the dielectric constants being 1 for the background and 13 for the rods [9]. For waveguides, taking advantage of a strictly TM photonic bandgap, only TM polarized modes may become guided, and consequently the waveguide will be extremely polarization sensitive (operating as a polarizer). TM bandgap guided modes, being localized to the line defect, are characterized by a frequency within the photonic bandgap of the surrounding photonic crystal. In particular, it is possible to guide light using a line defect with a low concentration of high-index material compared to the surrounding structure, and therefore the guiding mechanism, photonic bandgap guidance, is fundamentally different from conventional indexguiding waveguides. In this letter we consider the concentration of high-index material in the waveguiding region to be high compared to the surrounding structure for waveguide widths $W$ shorter than $1.0 \Lambda$. Consequently, only widths larger than $1.0 \Lambda$ are investigated in this paper.

The band diagram shown in Fig. 2 was obtained from calculations with a supercell height $H=W+9 \sqrt{3} \Lambda$, where the bands on either side of the bandgap are not completely dense. The dense bands shown in Fig. 2 correspond to an infinitely high supercell. In the case of an infinitely high supercell, it is

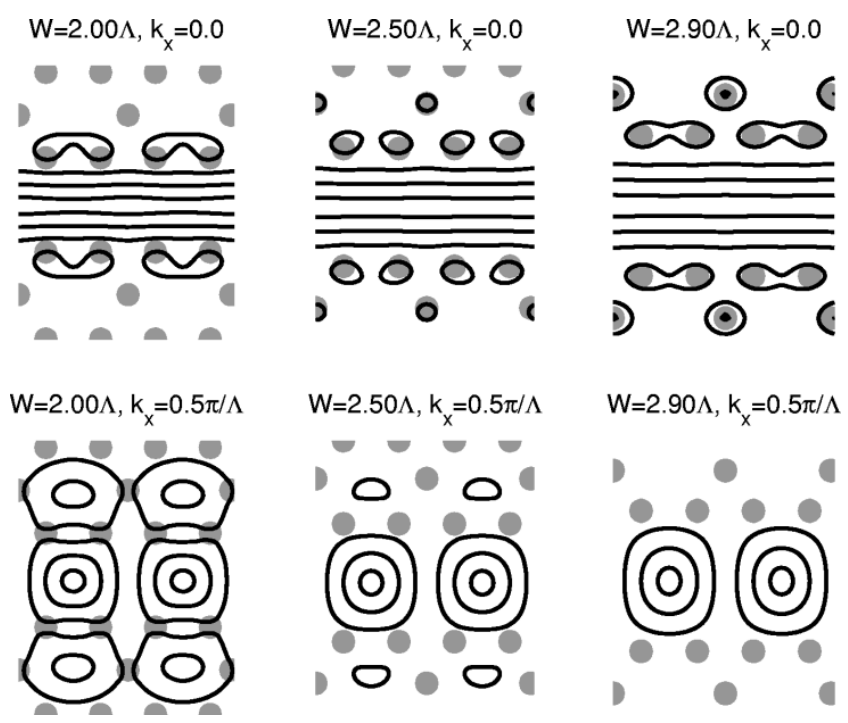

Fig. 4. Six field plots shows the dependency of localization and field structure upon the waveguide width $W$ and the $k_{x}$ value.

only necessary to probe the bandgap structure along the $k_{x}$-axis from zero to $\pi / 2 \Lambda$, because of the collapse of the irreducible Brillouin zone in the $k_{y}$-direction as $H$ tends toward infinity.

The guided modes of three different waveguide widths are shown in the bandgap. The monotone sweep of frequencies between $k_{x}=0$ and $k_{x}=\pi / 2 \Lambda$ means that only the $k_{x}=0$ and $k_{x}=\pi / 2 \Lambda$ points in the Brillouin zone need to be calculated in order to obtain the frequency intervals of guided modes for given waveguide widths. Fig. 3 shows these frequency intervals as a function of waveguide width $W$, where single-moded and dualmoded domains are shaded light and dark, respectively. This is interesting from a construction point of view, as single-moded waveguides are generally more useful than multi-moded waveguides.

With respect to the bandgap edges, Fig. 3 only shows the general picture, as the TM bandgap edges depend slightly upon $k_{x}$. The bandgap shown in Fig. 3 is the maximum bandgap valid for the complete set of $k_{x}$-values. The thin domain edge corresponds to $k_{x}=0$, while the thick edge corresponds to $k_{x}=\pi / 2 \Lambda$.

Using Fig. 3, it is possible to determine the characteristic parameters introduced in Fig. 1, if the frequency of the wave in the waveguide is given. Choosing a wavelength of $1550 \mathrm{~nm}$ (the standard wavelength of optical communications), a normalized frequency of 0.6 , and a waveguide width of $2.2 \Lambda$ as an example, a single guided mode far from the bandgap edges is obtained. In this example $\Lambda$ is $465 \mathrm{~nm}$, hence $D$ is $209 \mathrm{~nm}$, and thus the structure appears realizable [9].

Fig. 4 shows six field plots (the electric field amplitude squared) of modes from mode-domain II. It demonstrates that the modes are localized better far from the bandgap edges. This is observed by comparing Figs. 3 and 4: As the waveguide width increases, we know from Fig. 3 that mode-domain II is approaching the bandgap edge in the case of $k_{x}=0$. Fig. 4 shows a decreasingly localized mode. The same line of argument applies for $k_{x}=\pi / 2 \Lambda$, where modes in larger waveguides are better localized. 
Although changes in the waveguide width affect the localization of the modes for a given $k_{x}$, the field intensity distribution is almost unaffected until the modes becomes completely delocalized. The transition from a guided to an unguided mode will of course have an effect. With respect to variations in $k_{x}$, both the localization (as explained above) and the field structure are affected simultaneously. The field structures shown in Fig. 4 are clearly different for $k_{x}=0$ and $k_{x}=\pi / 2 \Lambda$. In fact, the field structure transforms continuously from one to the other as $k_{x}$ goes from $k_{x}=0$ to $k_{x}=\pi / 2 \Lambda$.

In conclusion, we have theoretically investigated a waveguide consisting of a line defect within a new fundamental hexagonal photonic bandgap material, the Kagomé structure. The investigation shows the Kagomé waveguide to have well-localized low-index-guided TM modes, and that the TM modes are better localized far from the bandgap edges. Furthermore, the frequency domain of the TM guided modes for small waveguide widths have been mapped out, allowing us to determine the number of guided modes in the waveguide for specific waveguide widths and frequencies.

\section{REFERENCES}

[1] E. Yablonovitch, "Photonic band-gap structures," J. Opt. Soc. Amer. B., vol. 10, no. 2, pp. 283-295, 1993.

[2] A. Mekis, J. C. Chen, I. Kurland, S. Fan, P. R. Villeneuve, and J. D. Joannopoulos, "High transmission through sharp bends in photonic crystal waveguides," Phys. Rev. Lett., vol. 77, no. 18, pp. 3787-3790, 1996.
[3] A. Mekis, S. Fan, and J. D. Joannopoulos, "Bound states in photonic crystal waveguides and waveguide bends," Phys. Rev. B., vol. 58, no. 8 , pp. 4809-4817, 1998.

[4] J. D. Joannopoulos, R. D. Meade, and J. N. Winn, Photonic Crystals, Molding the Flow of Light. Princeton, NJ: Princeton Univ. Press, 1995.

[5] H. Benisty, "Modal analysis of optical guides with two-dimensional photonic band-gap boundaries," J. Appl. Phys., vol. 79, no. 10, pp. 7483-7492, 1996.

[6] M. Plihal and A. A. Maradudin, "Photonic band structures of two-dimensional systems: The triangular lattice," Phys. Rev. B., vol. 44, no. 16, pp. 8565-8571, 1991.

[7] T. Søndergaard, J. Broeng, A. Bjarklev, K. Didri, and S. E. Barkou, "Suppression of spontaneous emission for a two-dimensional honeycomb photonic bandgap structure estimated using a new effective-index model," IEEE J. Quantum Electron., vol. 34, no. 12, pp. 2308-2313, 1998.

[8] D. Cassagne, C. Jouanin, and D. Bertho, "Photonic band gaps in a two-dimensional graphite structure," Phys. Rev. B., vol. 52, no. 4, pp. R2217-R2220, 1995.

[9] T. Hamano, H. Hirayama, and Y. Aoyagi, "New technique for fabrication of two-dimensional photonic bandgap crystals by selective epitaxy," $J$. Appl. Phys., vol. 36, pp. L286-L288, 1997.

[10] J. B. Nielsen, T. Søndergaard, S. E. Barkou, A. Bjarklev, J. Broeng, and M. B. Nielsen, "The two-dimensional Kagomé structure, a new fundamental hexagonal photonic crystal configuration," Electron. Lett., vol. 35, no. 20, pp. 1736-1737, 1999.

[11] R. D. Meade, A. M. Rappe, K. D. Brommer, J. D. Joannopoulos, and O. L. Alerhand, "Accurate theoretical analysis of photonic band-gap materials," Phys. Rev. B., vol. 48, pp. 8434-8437, 1993. 